\title{
Free operant avoidance behavior in hooded rats: IRTs and response chains
}

\author{
DELLA M. HANN and A. E. ROBERTS \\ Catawba College, Salisbury, North Carolina
}

\begin{abstract}
Twenty-five hooded rats were given 50 avoidance training sessions with leverpress IRTs obtained during as well as between sessions. Five qualitatively different shapes to the IRT profiles were found: single- or double-peaked shapes, positively or negatively accelerated shapes, and U-shaped distributions. Both the specific shape of the profile and the stability of that shape during a session were related to the avoidance proficiency of an individual rat. Six rats received two additional training sessions, with the final $30 \mathrm{~min}$ of each videotaped to obtain frequency counts of nine behavior categories. The observational data showed that avoidance-proficient rats typically incorporated the leverpress requirement into repetitive response chains.
\end{abstract}

Under the free operant avoidance procedure (Sidman, 1953), each criterion response postpones an electric shock for a fixed period (the R-S interval). If the response is not emitted, shock is given periodically (the $\mathrm{S}-\mathrm{S}$ interval). This schedule permits the subject to develop a rate of response that leads to relatively low frequencies of unavoided shock. Considerable attention has been given to the analysis of the behavior that develops under this avoidance schedule, and recently the examination of changes in avoidance behavior that might occur within a training session has been of particular interest.

For example, after the warm-up period (a repeated, temporary period of relatively poor avoidance at the outset of each session), changes in leverpress avoidance behavior correspond to the avoidance proficiency of the individual rat. Roberts (1978) used shock rate values to designate individual rats as good avoiders (low shock rates), nonavoiders (high shock rates), or poor avoiders (a broad range of rate values in between). The response and shock rates of individual nonavoiders and good avoiders were relatively stable over the post-warm-up period, but poor avoiders showed progressive improvements in these measures during the session, but not between sessions.

The picture of post-warm-up avoidance behavior may be somewhat different when using a different index of avoidance, the distribution of leverpress responses within the R-S interval (interresponse times: IRTs).

This paper is based, in part, on a thesis submitted by Della $\mathbf{M}$. Hann to the Department of Psychology in partial fulfillment of the requirements for Departmental Honors. Portions of the data were presented at the annual meeting of the Southeastern Psychological Association, New Orleans, 1982. This research was supported by a grant from the Grants and Research Committee of Catawba College awarded to A. E Roberts. Della M. Hann is now at the University of Tennessee. Reprints may be obtained from A. E. Roberts, Department of Psychology, Catawba College, Salisbury, NC 28144.
The monitoring of avoidance IRT data has diminished in recent years, but an early study by Wertheim (1965), using an R-S $=20 \mathrm{sec}$, reported changes in an IRT distribution during a session. The IRTs of three rats were brief $(0$ to $2 \mathrm{sec})$ in the first $20 \mathrm{~min}$ of a $60-\mathrm{min}$ session, but became more extended (6 to $14 \mathrm{sec}$ ) by the final $20 \mathrm{~min}$. That is, IRT distributions typically were U-shaped early, but a "peak" within the distribution appeared later in the session. These IRT data could represent a lessening in response rate as the session progressed. The Roberts (1978) data, however, suggest that the picture of post-warm-up avoidance offered from the IRT data of Wertheim may be incomplete in that a given IRT profile should be considered within the context of the avoidance proficiency of the rat producing it. The avoidance behavior of a poor avoider improves during a session, so a rat from this population would be expected to generate an IRT profile that also changed during that session. Stated differently, changes in IRT profiles over the post-warm-up period may not correlate so much with "time" (as suggested by the Wertheim data) as with "avoidance improvement," and these two variables are most likely to be confounded for the poor avoider. On the other hand, the level of avoidance proficiency of the good avoider and nonavoider is established early in the session, and postwarm-up changes in the IRT profiles of these rats might not be found.

A correspondence between avoidance proficiency and IRT distribution seems plausible, but experimental support for such an association actually is restricted because of the limited collection of individual IRT profiles in the literature. Individual IRT profiles that are available (cf. Sidman, 1966) usually appear to assess the influences of a separate treatment condition, and grouped IRT profiles, while available (Weissman, 1962), obscure the interanimal diversity in the data that probably is present.

Indeed, a number of basic questions about avoidance 
IRT profiles have yet to be addressed. First, and perhaps most obvious, can a correspondence between specific IRT profiles and the degree of avoidance proficiency be demonstrated? Second, at what point in training does a rat produce a reliable IRT profile, that is, early in training or after an extended number of sessions? This question was raised originally by Anger (1963), who was unable to draw upon the literature of the time for an answer. Third, does the shape of an IRT profile undergo changes during an avoidance session?

The experiment described below addressed these questions by giving 5090 -min avoidance training sessions to 25 rats and collecting IRT data from each rat. We anticipated that the levels of avoidance proficiency would vary among the rats in the sample (i.e., good avoiders, poor avoiders, and nonavoiders) and that we could compare given levels of proficiency to the specific IRT profiles that developed (Question 1). IRT data from these 50 sessions would serve to document the evolution of these profiles as well as the stability of those profiles across sessions (Question 2). Finally, the IRT data were recorded at 30-min intervals during each of the final 10 training sessions to monitor the within-session stability of the individual profiles (Question 3).

As the experiment progressed, a fourth question emerged. The leverpress response has been viewed as being incompatible with natural species-specific defense reactions, SSDRs (Bolles, 1970, 1971), but rats do learn to activate the lever to postpone shock and can do so proficiently. Our fourth question was whether the "unnatural" leverpress becomes incorporated into a broader stream of activities, perhaps to be more compatible with "natural" SSDRs. This question was addressed by observing the activities of selected rats during terminal portions of later training sessions when avoidance measures were stable.

\section{METHOD}

Subjects

Twenty-five female hooded rats, obtained from Blue Spruce Farms, New York, served as subjects. The rats were experimentally naive and weighed about $160 \mathrm{~g}$ at the beginning of the experiment.

\section{Apparatus}

Three LVE operant chambers (Model 143-20), measuring $30 \times 24 \times 26 \mathrm{~cm}(1 \times \mathrm{w} \times \mathrm{d})$, were used. Each chamber was modified by the placement of a houselight in the center of the plastic ceiling and the removal of all standard assemblies protruding into the chamber. The floor grids were brass rods $(0.25 \mathrm{~cm}$, diam) spaced $1.3 \mathrm{~cm}$ apart and parallel to the width of the lever. A $2.5 \times 3 \mathrm{~cm}$ lever extended $2.5 \mathrm{~cm}$ into each chamber from the middle of the intelligence panel with the top surface of the lever $6 \mathrm{~cm}$ above the grid floor. A deadweight of $36 \mathrm{~g}(0.36 \mathrm{~N})$ was required to operate each lever. A $1-\mathrm{mA}$ 0.3 -sec shock was delivered to the grids, lever, and metal sides of the chamber from a constant-current shock generator (BRS, SG-901) via a scrambler (BRS, SC-901). Each chamber was placed in a sound-attenuated box with a blower fan providing ventilation and background noise $(76 \mathrm{~dB}$ SPL). The electromechanical programming and recording equipment was in an adjoining room.

\section{Procedure}

Each rat was trained under a free operant avoidance schedule under which each leverpress postponed shock for $15 \mathrm{sec}$ (R-S interval) and briefly $(0.3 \mathrm{sec})$ turned off the houselight. The houselight otherwise was on throughout the session. In the absence of a leverpress, shock was given once every 5 sec (S-S interval). A total of 5090 -min sessions were given, although, as noted below, six rats were discontinued after 20 sessions.

The number of responses and unavoided shocks in each session were recorded automatically. Response frequencies also were channeled into one of 10 consecutive 1.5 -sec bins of the R-S interval (the IRT measure) as well as into a bin at the end of the R-S interval ("shock-produced" responses). These data were recorded at the end of each session, but also were collected every $30 \mathrm{~min}$ over the final 10 training sessions (Sessions 41-50).

Six rats were selected for two additional training sessions, with the final $30 \mathrm{~min}$ of each videotaped. Initial observations of

Table 1

The Behavior Categories, Symbols, and Descriptions Followed in Recording the Avoidance Behaviors of the Six Rats

\begin{tabular}{cl}
\hline Recording Symbol & Behavior Category \\
\hline BP & Back Paw Response \\
CR & Crouch Position \\
J & Jumping Response \\
LH & Lever Hold Response \\
U & Upward Movement Response \\
P & Prone Position \\
ST & Standing Position \\
T & Turn Response \\
W & Walking Response
\end{tabular}
Behavior Description

Keeping the lever continually depressed with a back paw

The rat in a relatively immobile, semierect position while not in contact with the lever

A jumping response in which all four paws are out of contact with the grids Keeping the lever continually depressed with one or two forepaws

The rat briefly raising its upper torso

The rat in a relatively immobile position with all four paws in contact with the grids The rat in a relatively immobile position of standing erect

Rotating body position by at least $90 \mathrm{deg}$

A locomotor response resulting in a change in location in the chamber 
these tapes yielded the behavioral categories identified and described in Table 1. The videotapes were reviewed (by D. M. H.) to obtain frequency counts of each category as well as to note its position within an activity sequence. Activities elicited by unavoided shocks were not counted. The videotapes were viewed again (by A. E. R.) to verify the accuracy of the written records for each rat,

\section{RESULTS}

\section{Avoidance Acquisition}

Considerable interanimal differences in avoidance proficiency were present within the sample. For descriptive purposes, each rat was classified as being a good avoider, a poor avoider, or a nonavoider (GA, PA, NA, respectively), based on mean shock-rate values from Sessions 16-20: less than $1 \mathrm{~min}$ (GA), 1-2.5 min (PA), and greater than $2.5 \mathrm{~min}$ (NA). Table 2 summarizes the avoidance data for the three groupings of rats. The responding of the six NA rats was primarily shock-produced, so these rats were dropped from the experiment. Three of the nine PA rats lessened shock rate after Session 20, but none of them to the level of meeting our criterion for a good avoider. The shock rate of each of the other six PA rats had decreased rapidly early in training, leveled temporarily, and then increased. For example, the mean shock rate of Rat 32 was 1.1 min over Sessions 6-10 but increased to 2.0 (Sessions 16-20) and remained at that level for the remainder of training. Finally, neither the shock rate nor the response rate values of any GA rat changed substantively after Session 20.

\section{IRT Profiles: Over Sessions}

One dimension to our experiment was to document the various IRT profiles that developed over sessions. Five qualitatively different shapes in IRT distributions among the 25 rats were found, and each is illustrated in Figure 1. Three shapes were found from the GA rats, with the most common having a single peak within the R-S interval (six rats, cf. Rats 38, 47, and 53). Figure 1 (the final block of sessions) shows that the exact location of this peak varied somewhat among rats. A second shape (two rats, cf. Rat 44) contained a double peak within the R-S interval. In the third shape, a peak to the

Table 2

Mean Response and Shock Rates for the Rats After Being Designated Good, Poor, or Nonavoiders (GA, PA, NA, respectively)

\begin{tabular}{lccc}
\hline & $\begin{array}{c}\text { Group GA } \\
(\mathrm{N}=10)\end{array}$ & $\begin{array}{c}\text { Group PA } \\
(\mathbf{N}=9)\end{array}$ & $\begin{array}{c}\text { Group NA } \\
(\mathbf{N}=6)\end{array}$ \\
\hline Mean & 12.5 & Responses/Min & \\
SD & 1.8 & 9.7 & 8.3 \\
Range & $10.1-16.1$ & 1.9 & 1.1 \\
& \multicolumn{4}{c}{ Shocks/Min } \\
Mean & 0.59 & 1.63 & $7.3-10.3$ \\
SD & 0.19 & 0.25 & 3.08 \\
Range & $0.36-0.74$ & $1.25-1.90$ & 0.45 \\
\hline
\end{tabular}

Note-Group means were based on values obtained for each of the rats from training sessions 16-20. profile was absent (two rats, cf. Rat 33 ) as the distribution was positively accelerated, reflecting relatively short-latency responses.

The fourth general shape was negatively accelerated (i.e., responses tended to occur relatively late in the R-S interval) and was found for six PA rats (cf. Rat 42). The profiles of the other three PA rats are not presented in Figure 1 as they were condensed versions of the positively accelerated shape already noted, that is, were similar to that for Rat 33 in Block 2. The fifth profile was U-shaped (relatively few responses within the R-S interval) and was obtained for each of the six NA rats. These profiles also are not given in Figure 1, because each was similar to the one given for Rat 53 in Block 1.

Two other points of interest are noteworthy in Figure 1. First, the IRT profiles obtained in the early train ing sessions (Blocks 1 to 3 ) generally did not serve to isolate rats that later developed an effective avoidance response rate from those that did not. Second, peaks to IRT profiles of GA rats appeared neither suddenly nor early in training. These peaks generally emerged in Blocks 4 or 5 , well after their response and shock rate values had stabilized (the profile for Rat 44 in Block 1 was atypical in this respect).

\section{IRT Profiles: Changes During Sessions}

Another dimension to our study of avoidance IRTs centered on changes in IRT profiles that might occur within a session. We addressed this issue by recording IRT and shock frequency data from each rat every 30 min during the final 10 training sessions. Two sets of IRT profiles then were prepared from these measures, with one representing data from the three consecutive 30-min periods of a session. The second set was organized on the basis of shock rate values obtained from each 30-min period to separate IRTs present when the shock rate was $0-0.99,1.00-1.99$, or $2.00-2.99$. Both sets of profiles for selected rats appear in Figure 2. Thus, one set of IRTs ("time based") show profiles over the three consecutive 30-min periods of a session regardless of avoidance proficiency within those periods; the other set of IRTs ("shock rate based") give profiles as a function of avoidance proficiency rather than time in the session. The numbers within each panel give the mean shock rates in the respective measurement periods.

Considering the time-based IRTs, a change in the shape of the profiles of GA rats (the top six sets of panels: Rats $36,38,40,48,50$, and 53 ) was most likely between the first and second $30 \mathrm{~min}$ of the session. The U-shaped patterns that many had developed within the first $30 \mathrm{~min}$ had disappeared by the second $30 \mathrm{~min}$. Those profiles present in the second 30 -min period, however, were maintained into the final $30 \mathrm{~min}$. The IRT profiles of two GA rats (cf. Rat 48) remained unchanged over each of the three periods. On the other hand, the profiles of the six PA rats that showed the negatively accelerated shape (cf. Rats 35 and 42) changed in each 30 -min period, and the appearance of a peak within the 

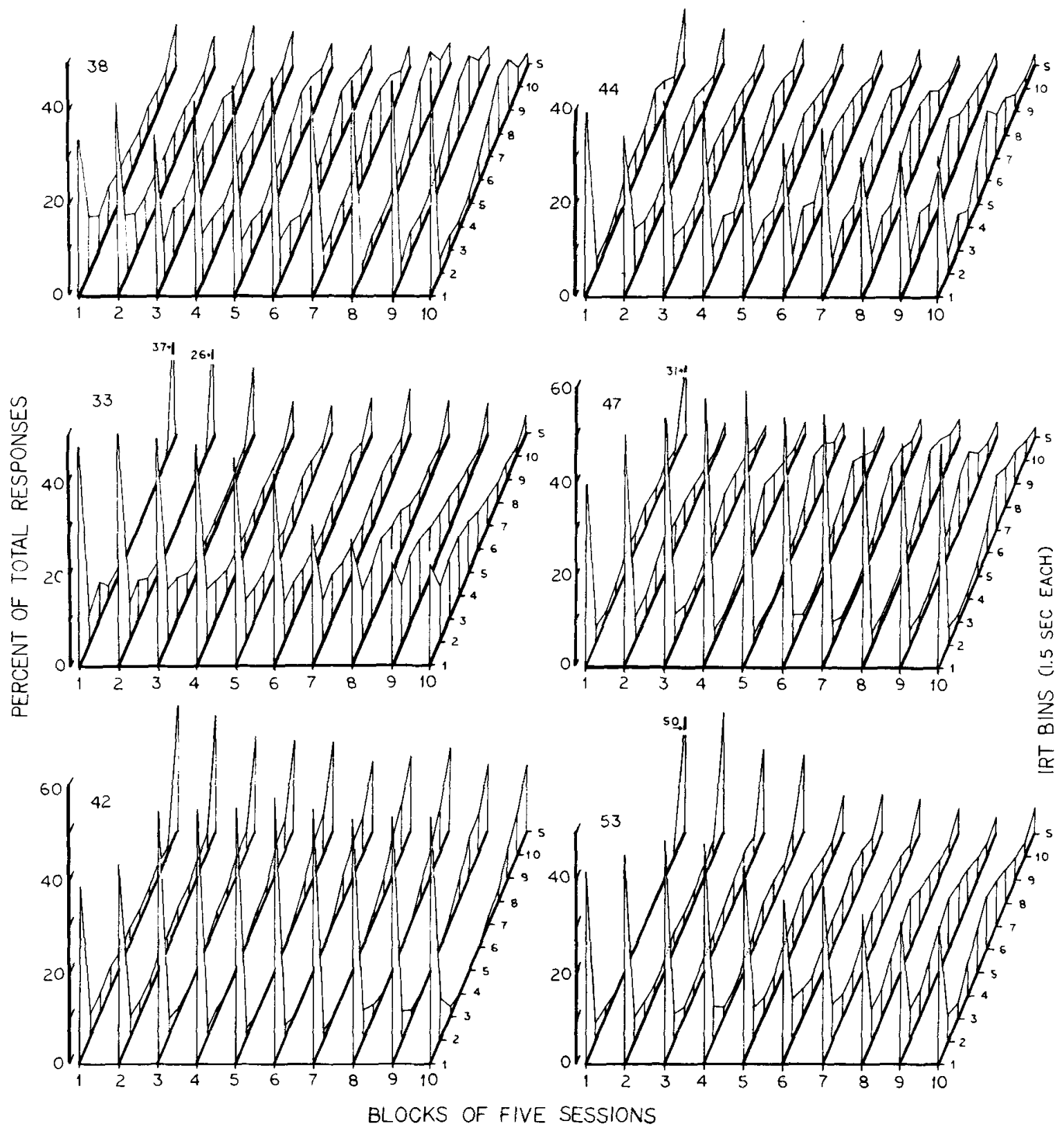

Figure 1. IRT distributions of selected rats over the 50 avoidance training sessions (presented in blocks of five sessions). Responses after the $R-S$ interval had elapsed were channeled into bin $S$, that is, shock-produced responses.

distribution was not uncommon later in the session. The profiles of the other three PA rats retained an abbreviated, positively accelerated shape throughout the session.

The shock-rate-based IRT data show that the shape of the IRT profile generally varied as a function of avoidance proficiency. Specifically, U-shaped IRTs were associated with relatively high shock rates with a peak in the profile emerging when shock rate was relatively low. This correspondence is most noticeable when the two sets of panels for the PA rats (35 and 42) are compared: IRTs associated with relatively high, medium, and low shock rates (right-side panels) were similar in shape to those in the first, second, and third 30 -min periods, respectively (left-side panels).

\section{Avoidance Behavior Sequences}

At the completion of the 50 training sessions, five GA rats and one PA rat were given two additional sessions. The videotapes from the final $30 \mathrm{~min}$ of these sessions were reviewed to obtain the frequencies of the behavior categorized in Table 1. The five GA rats were selected soley on the basis of having developed different IRT pro- 

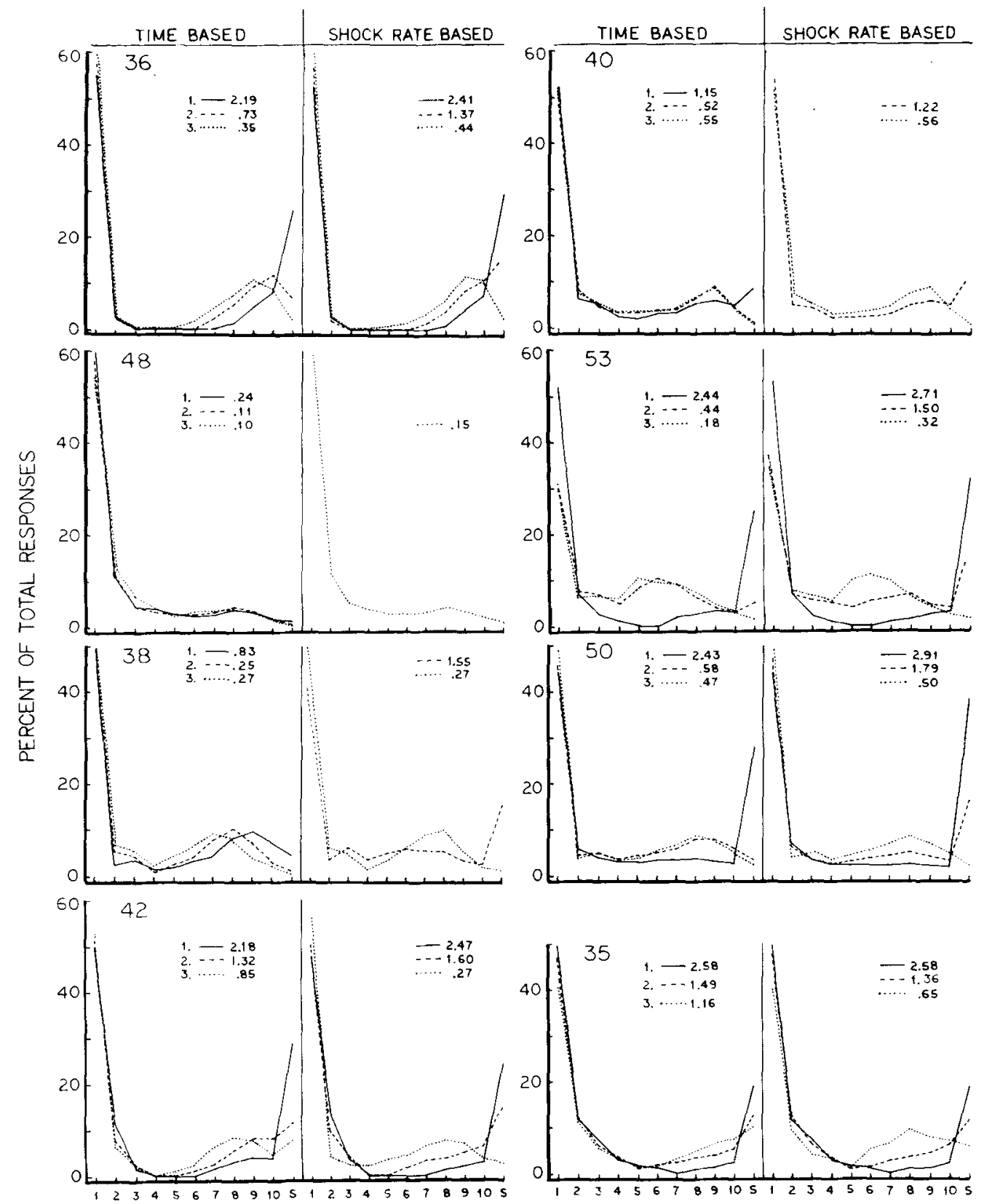

IRT BINS (1.5 SEC EACH)

Figure 2. Left-side panels (time based) present IRT profiles obtained from three consecutive 30-min periods of each of the final 10 training sessions. Right-side panels (shock rate based) show IRT profiles as a function of the avoidance proficiency within each of the separate 30 -min periods. See text for additional details.

files. The avoidance measures of these rats are summarized in Table 3 . We found that a given leverpressing episode often involved multiple lever activations. Emphasis thus was given to enumerating those activities that occurred between consecutive leverpressing episodes (i.e., present during the R-S interval) rather than each discrete leverpressing instance. Table 4 shows that most typical sequences in terms of the percentage of the total number of activity sequences counted for that rat. Fig- ure 3 gives a more detailed analysis of these sequences by presenting the data in terms of the conditional probabilities for each activity that occurred between consecutive leverpressing episodes. The IRT profiles for each rat also appear in Figure 3.

Rat 33. Most of the activities that involved lever activations were associated with leverholding $(\mathrm{LH})$. This rat either interrupted leverholding with lever activations or simply released the lever, briefly paused (CR), and 
Table 3

Mean Response and Shock Rates for the Six Rats Selected for Observation

\begin{tabular}{lrrrrr}
\hline & \multicolumn{2}{c}{$\begin{array}{c}\text { Mean } \\
\text { Resp/Min }\end{array}$} & & \multicolumn{2}{c}{$\begin{array}{c}\text { Mean } \\
\text { Shocks/Min }\end{array}$} \\
\cline { 2 - 3 } & Tr & Obs & & Tr & Obs \\
\hline Rat 32 & 10.0 & 10.6 & & 0.35 & 0.30 \\
Rat 38 & 10.4 & 10.1 & & 0.50 & 0.33 \\
Rat 43 & 9.3 & 10.1 & & 0.52 & 0.46 \\
Rat 44 & 9.5 & 9.6 & & 0.47 & 0.44 \\
Rat 47 & 9.9 & 10.3 & & 0.38 & 0.21 \\
Rat 42 & 9.7 & 9.4 & & 1.37 & 1.44 \\
\hline
\end{tabular}

Note-Sessions 46-50 provide the basis for training means; the two 30-min videotaping periods provide the basis for observation means. Means are given for the final five training (Tr) and the two observation (Obs) sessions.

Table 4

Activity Sequences Expressed in Terms of the Percentage of the Total Number of Sequences Recorded for a Given Rat

\begin{tabular}{|c|c|c|c|}
\hline Activities & $\%$ & Activities & $\%$ \\
\hline \multicolumn{2}{|c|}{ Rat 33} & \multicolumn{2}{|c|}{ Rat 38} \\
\hline LH-R & 31 & W-P-W-T-R & 48 \\
\hline LH-CR-R & 26 & BP-R & 12 \\
\hline BP-R & 12 & LH-R & 9 \\
\hline BP-var & 10 & W-P-W-T-vat & 9 \\
\hline CR-R & 5 & BP-W-P-W-T-R & 5 \\
\hline LH-var & 4 & LH-W-P-W-T-F & 3 \\
\hline $\mathbf{S}$ & 5 & $\mathbf{S}$ & 6 \\
\hline Total & 93 & Total & 92 \\
\hline \multicolumn{2}{|c|}{ Rat 43} & \multicolumn{2}{|c|}{ Rat 44} \\
\hline ST-BP-R & 32 & BP-R & 67 \\
\hline BP-R & 29 & BP-U-T-R & 8 \\
\hline ST-R & 16 & BP-W-P-W-T-R & 7 \\
\hline BP-P-W-U-T-J-R & 14 & W-P-W-T-var & 6 \\
\hline W-P-W-U-T-J-R & 2 & BP-U-R & 5 \\
\hline $\mathbf{S}$ & 4 & $\mathrm{~S}$ & 3 \\
\hline Total & 97 & Total & 95 \\
\hline \multicolumn{2}{|c|}{ Rat 47} & \multicolumn{2}{|c|}{ Rat 42} \\
\hline P-U-T-J-R & 82 & BP-R & 13 \\
\hline BP-U-J-R & 7 & BP-T-R & 9 \\
\hline BP-R & 6 & BP-U-T-R & 8 \\
\hline$S$ & 4 & $\mathrm{~S}$ & 60 \\
\hline Total & 99 & Total & 90 \\
\hline
\end{tabular}

Note-A leverpress is noted by an $R$, and an $S$ indicates that a sequence terminated in unavoided shock. Other symbols are as described in Table 1. The "var" designations reflect the combination of separate low-frequency variations in an otherwise high-frequency category or sequence.

then leverpressed. The BP sequences began while leverholding. The rat stepped on and then over the lever (without activating the microswitch) into a BP; the lever then was activated by a back paw. The BP changed into an $\mathrm{LH}$ as the rat either backed over the lever or stepped down from the lever, turned, and returned to leverholding. The absence of a peak in the IRT profile (see Figure 3) complements our observations that the rat was almost continually engaged in one of these activities, that is, with a minimal number of pauses between activities.

Rat 38. The activity sequences of Rat 38 were more stereotypic and involved the rat's being away from the lever. Most of the activities involved a W-P-W-T sequence: The rat stepped on the lever and over it, and then walked a few steps away from it. After pausing (facing the rear wall of the chamber, parallel to a side wall), the rat backed up one or two steps, turned toward, and then stepped on and over the lever. Thus, the leverpressing episode of one sequence led to the $W$ observed for the subsequent sequence. The $\mathrm{LH}$ and $\mathrm{BP}$ activities that emerged as the "walks" over the lever were not completed.

Rat 43. Three sets of leverpressing sequences were observed for Rat 43 . In one, the rat simply shifted or "adjusted" its position on the lever while in a BP and thereby activated the microswitch. The second was a variation of the first. The rat raised into a standing position, with a back paw remaining on the lever; this movement activated the microswitch. After a brief pause, the rat returned to a BP, again activating the microswitch. The third activity (P-W.U.T-J) was more sequentially organized and, in contrast to the other two, did not involve physical contact with the lever. The rat stepped off and away from the lever (usually from a BP) and paused in a prone position. The rat then backed, turned, and jumped over the lever (without lever contact). The lever was activated as the rat stepped on and across the lever in to a BP.

Rat 44. This rat also showed activity sequences that involved physical contact with the lever and that occurred away from the lever. The former consisted of the rat's facing a rear corner of the chamber and, while maintaining BP contact with the lever, stretching its body almost full length, pausing, and backing up either to emit a subsequent stretching movement or to face a corner adjacent to the lever. In this second instance, the rat stepped from the lever, turned, and returned to BP contact with the lever. In either case, the return-fromthe-stretch movements produced multiple lever activations. The W-P-W-T sequence, present when the rat's stretching movements seemingly "pulled it off" the lever, was similar to that described for Rat 38.

Rat 47. The most frequent sequence of Rat 47 (P-U-T-J) was similar to that described for Rat 43, except that this rat did not walk from the lever but simply stepped away from BP contact. As with Rat 43, the turning motion was always clockwise and the jump was always over the lever to the opposite side of the chamber.

Rat 42. This rat served as a representative of the PA subgroup, and most of the observed activities terminated in unavoided shock. In most cases, the rat was in physical contact with the lever prior to shock delivery-either a BP (35\%) or an LH (17\%). Those activities that involved lever activation and postponed shock were associated with a BP; that is, they were consequent either to 

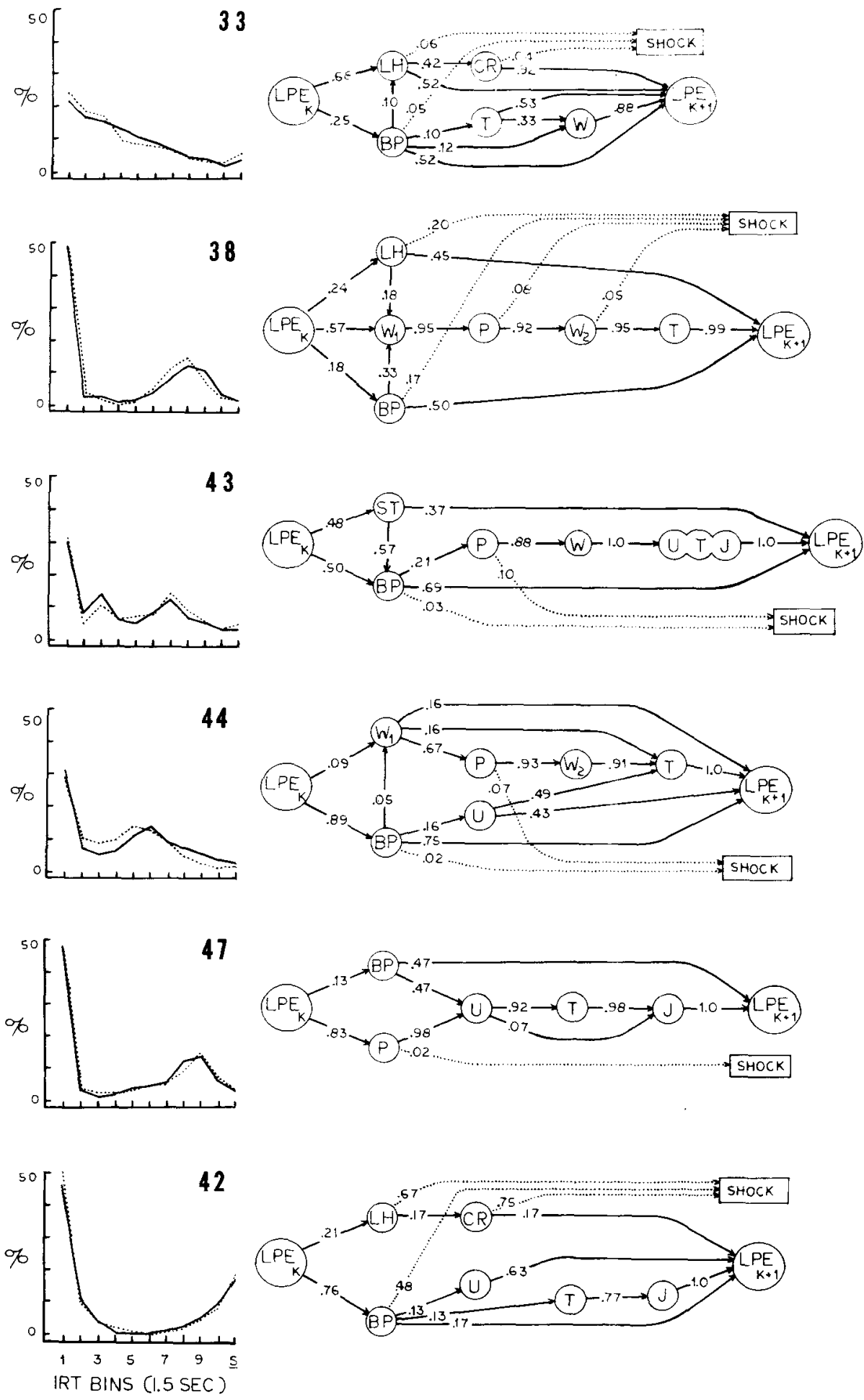

Figure 3. The conditional probabilities of each activity shown by each of the six rats between consecutive leverpressing episodes, $\mathbf{L P E}_{k}$ and $L P E_{k+1}$. The symbols are identified in Table 1. Low-probability behaviors have been omitted for clarity, so the sum of the probabilities at each fork may be less than 1.0. IRT distributions compare the IRT profiles of the observation periods (solid lines) with those from the final five training sessions (broken lines). 
an adjustment of the rat's body on the lever (in the manner described for Rat 43 or to a 180-deg turn that changed the BP position.

\section{DISCUSSION}

The present experiment attempted to answer four general questions about avoidance IRTs. One question centered on a possible correspondence between a rat's avoidance proficiency and its IRT profile. Three levels of avoidance proficiency of individual rats were used in this study (our designations of good avoiders, poor avoiders, and nonavoiders) based on the rate of unavoided shock. Five qualitatively different shapes to IRT profiles were obtained during the experiment: profiles that had either a single or a double peak, profiles that were either positively or negatively accelerated, and U-shaped.

The single- and double-peaked profiles were obtained only from good avoiders (i.e., rats having relatively low shock rates) although some GA rats did produce a positively accelerated profile. The rats with relatively high shock rates (the nonavoiders) consistently showed a Ushaped profile that indicated only minimal responding within the R-S interval. Finally, PA rats, whose shock rates spanned the middle ranges, tended to produce an IRT profile that was negatively accelerated, although an abbreviated positively accelerated shape was found for some PA rats. In short, only the GA rats generated an IRT profile containing a peak, but no one single profile was characteristic of these rats whether one considers the general shape (three were obtained) or the specific location of the peak. Obviously, any one of a number of temporal strategies can be brought to bear to successfully execute the shock-postponement requirement.

Our second question concerned when in training a rat began to produce a consistent IRT profile. Our answer to this question is less definitive as the degree of avoidance proficiency contributes to the stability of the profile. For example, the NA rats presented a U-shaped profile in the initial training sessions, and these remained unchanged over the 20 sessions given these rats. The profile from a GA or PA rat typically evolved gradually over sessions. A given shape began to appear consistently after response- and shock-rate values became asymptotic. Once established, that pattern remained relatively stable across sessions.

The negatively accelerated profiles obtained from the PA rats bear directly on our third question, namely, whether the IRT profiles were stable within a session. These profiles were indicative of avoidance behavior that became progressively more proficient during the session. These IRTs were based on data collected at the end of the session and actually were a composite of two qualitatively different shapes (a U-shape and a peak shape: see Figure 2), each correlated with a different level of avoidance proficiency. Anger (1963) also recognized this circumstance, since he argued that such profiles reflected the influence of "inhomogeneous be- havior." Most GA rats also showed periods of marginal shock avoidance and U-shaped profiles, early in the session, but this period was transitory (i.e., a finite warmup period) and had less influence on the aggregate shape to the profile of that session. The profiles produced by GA rats were quite stable over the post-warm-up period. In short, once the avoidance response rate in a session becomes established and substantive reductions in shock rate unlikely, changes in the IRT profile also are unlikely.

Another feature to the IRTs (seen in Figure 2) was the large proportion of short-latency responses present in bin 1 (well over 50\% for most rats). One source for these responses was unavoided shock, which usually was followed by multiple, short-latency leverpresses (the "response burst") that offer minimal shock-postponement value (cf. Boren, 1961). The first of the set of responses were channeled into bin $S$ and the remainder into bin 1 . These shock-produced response bursts likely account for the large proportion of bin 1 responses recorded for the PA and NA rats. On the other hand, the high frequency of bin 1 responses for the GA rats was accompanied by low bin $S$ frequencies. This combination suggests that unavoided shocks did not contribute substantively to bin 1 frequencies. We began observing the rats and found that the act of postponing shock often involved a set of lever activations, for example, multiple leverpresses per episode, stepping on and across the lever, and shifts in body position while in contact with the lever. Moreover, these lever activations often appeared within a broader set of what seemed to be recurring activities. Anger (1963, pp. 493-495) discussed the possibility that free operant avoidance performance involved the emission of response chains, but he discounted their importance because, in part, of the lack of empirical evidence for such chains. Our informal observations suggested the opposite conclusion and led to our fourth question, namely, whether the rats showed a sequentially organized response topography.

We were surprised to discover that the GA rats showed a large repertoire of activities that involved physical contact with the lever as well as behaviors away from the lever. Considering the former, a number of activities centered on maintaining physical contact with the lever, only one of which was leverholding. Leverholding was observed for all GA rats, but this activity was not static and, while not monitored in this experiment, probably involved lever movements such as those recently documented by Davis (1981). Turning to the latter, we identified a number of locomotive actions that were organized in to highly repetitive response sequences. We might add that our observations of those GA rats not videotaped, as well as of rats trained subsequent to this experiment, confirm the presence of a variety of repetitive response chains, although a W.P.W-T.R sequence is quite common.

The intent of an IRT distribution is to isolate the locations of the avoidance response within the R-S interval, but an emphasis on the criterion response (e.g., the 
leverpress) as a discrete and singular event, could present a misleading picture about the nature of free operant avoidance behavior. We suggest a view of avoidance leverpressing as being emitted within a context of a broad sequence of activity, a response chain. The peak present in an IRT profile indicates the temporal location only of one element in the response chain, the one that fulfills the shock-postponement requirement. Given the organized nature of these response chains (e.g., W.P.W-TBP-W-P.W-T-BP-W-P. . .), a focus on the criterion response as the terminal link could direct attention away from other important components of the chain.

The presence of response chains, regardless of topography, typified rats that came to postpone shock proficiently as opposed to rats that did not. While all rats in our study did learn to make contact with the lever (e.g., pressed following unavoided shock), not all rats learned to use the lever to postpone shock. To state the obvious, the appearance of the latter does not necessarily evolve from the presence of the former. The rat can spend a large proportion of a session engaged in leverholding (Hurwitz, 1967), but this activity can interfere with avoidance acquisition, since the rat also must learn to release the lever so that it can be pressed again (an act the NA rats failed to learn). It is interesting to note in this regard that the amount of leverholding shown by rats can be increased (Davis \& Burton, 1976) or reduced (Meltzer \& Tiller, 1979) if so required by the avoidance contingency.

We will speculate that the response chains that the GA rats developed served to reduce leverholding by leading the rat away from the lever. Regardless of the specific topography, these chains represent, in our view, an accommodation between the necessity to avoid shock and the "unnatural" means to do so programmed by the experimenter. The NA and PA rats, on the other hand, seemed to approach the free operant avoidance task as if only a discrete and singular act (the leverpress) were required. Repetitive response chains were absent from their repertoires. From our view, the negatively accelerated IRT profiles found for several of the PA rats reflected a repertoire that was expanding beyond stereotypic leverholding, albeit of the LH or BP variety.

\section{REFERENCES}

ANGER, D. (1963). The role of temporal discriminations in the reinforcement of Sidman avoidance behavior. Journal of the Experimental Analysis of Behavior, 6, (Supplement), 477-506.

Bolles, R. C. (1970). Species-specific defense reactions and avoidance learning. Psychological Review, 77, 32-48.

Bolles, R. C. (1971). Species-specific defense reactions. In F. R. Brush (Ed.), A versive conditioning and learning (pp. 183-233). New York: Academic Press.

Bonen, J. J. (1961). Isolation of post-shock responding in a free operant avoidance procedure. Psychological Reports, 9, $265-266$.

Davis, H. (1981). The functional properties of leverholding during free operant avoidance. Behavior Analysis Letters, 1, 17-26.

Davis, H., \& Burton, J. (1976). Reinforcement of leverholding by avoidance of shock. Bulletin of the Psychonomic Society, 5, 617-620.

Hurwitz, H. M. B. (1967). Leverholding under free operant avoidance. Journal of the Experimental Analysis of Behavior, 10, 551-554.

Meltzer, D., \& Tillen, J. E. (1979). Bar press and bar release as avoidance responses. Journal of the Experimental Analysis of Behavior, 31, 373-381.

Roberts, A. E. (1978). Free operant avoidance in hooded rats: Some notes on within- and between-session stability. Psychological Record, 28, 253-262.

Sidman, M. (1953). Two temporal parameters of the maintenance of avoidance behavior by the white rat. Journal of Comparative and Physiological Psychology, 48, 444-450.

Sidman, M. (1966). Avoidance behavior. In W. K. Honig (Ed.), Operant behavior: Areas of research and application (pp. 172-217). New York: Appleton-Century-Crofts.

Weissman, A. (1962). Nondiscriminated avoidance behavior in a large sample of rats. Psychological Reports, 10, 591-600.

Wertheim, G. A. (1965). Some sequential aspects of IRTs emitted during Sidman avoidance behavior in the white rat. Journal of the Experimental Analysis of Behavior, 8, 9-15.

(Manuscript received June 1, 1983; revision accepted for publication October $7,1983$. ) 